\title{
Electrochemical oxidation of double-stranded polybisnorbornenes containing linearly aligned ferrocene linkers $\dagger$
}

\author{
Cheng-Lan Lin, Hui-Chun Yang, Nai-Ti Lin, I-Jui Hsu, Yu Wang* and \\ Tien-Yau Luh*
}

Received (in Cambridge, UK) 27th May 2008, Accepted 16th June 2008

First published as an Advance Article on the web 30th July 2008

DOI: $10.1039 / b 808958 b$

\begin{abstract}
Seventy percent of the ferrocene moieties in double-stranded polybisnorbornenes containing linearly aligned ferrocene linkers are oxidised and each of the neighbouring monomeric units in these polymers may strongly interact with each other; the oxidised form of $3 \mathrm{c}$ has been shown to be antiferromagnetic.
\end{abstract}

There has been ever burgeoning interest on the electrochemistry of macromolecules containing multiple electroactive centers. ${ }^{1-4}$ Multielectron transfer is common in these polymers and their voltammetric curves are occasionally similar to those of one-electron transfer reactions. ${ }^{1}$ The electrochemical behaviour of these polymers depends on whether interactions between these electroactive groups are present or not. ${ }^{1,2}$ For example, in the oxidation of poly(vinylferrocene) $\mathbf{1}$, all the ferrocene groups are oxidised at one potential and it is believed that there is little interaction between these iron-containing sandwich pending groups. ${ }^{1}$ On the other hand, when the ferrocene moieties are part of the polymeric backbone separated by different types of linkers, two reversible oxidation waves are in general observed. ${ }^{2}$ Similar behaviour is also found in ferrocene-containing dendrimers. ${ }^{3}$ We recently reported the first DNA-like double-stranded bispolynorbornenes 2 using covalent ferrocene linkers. ${ }^{5}$ The spacing occupied by each of the monomeric unit is about $5.5 \AA$ based on scanning tunneling microscopic images of $\mathbf{2}^{5}$ and on the $\mathrm{X}$-ray structure of a related monomer. ${ }^{6}$ Unlike those ferrocene-containing polymers in the literature, which are somewhat fluxional, $\mathbf{2}$ is more rigid and the ferrocene moieties in $\mathbf{2}$ are aligned essentially in eclipsed manner with a fixed distance. It is envisaged that interactions between the ferrocene units in 2 might be reflected by their electrochemical behaviour.

Since the oxidation potentials of the ferrocenedicarboxylate and the 4-aminobenzyl moieties in $\mathbf{2}$ were very close, and the 4-aminobenzyl moiety in $\mathbf{2}$ would be very labile and susceptible to hydrolysis, ${ }^{7}$ we decided to tackle the electrochemistry of 3 containing a linear array of ferrocene derivatives. The ferrocene moiety and 4-aminobenzoate groups in $\mathbf{3}$ would show very different oxidation potentials. In addition, the aminobenzoate moiety would be more stable than the aminobenzyl entity under various conditions. The syntheses of $\mathbf{3}$

Department of Chemistry, National Taiwan University, Taipei,

Taiwan 106.E-mail:tyluh@ntu.edu.tw;wangyu@ntu.edu.tw;

Fax: +886-2-2364-4971; Tel: + 886-2-2363-6288

$\dagger$ Electronic supplementary information (ESI) available: Details of electrochemical experiments and frontier orbitals from the DFT calculations. See DOI: 10.1039/b808958b essentially followed a similar strategy as described for $2^{5}$ and the details will be described elsewhere.

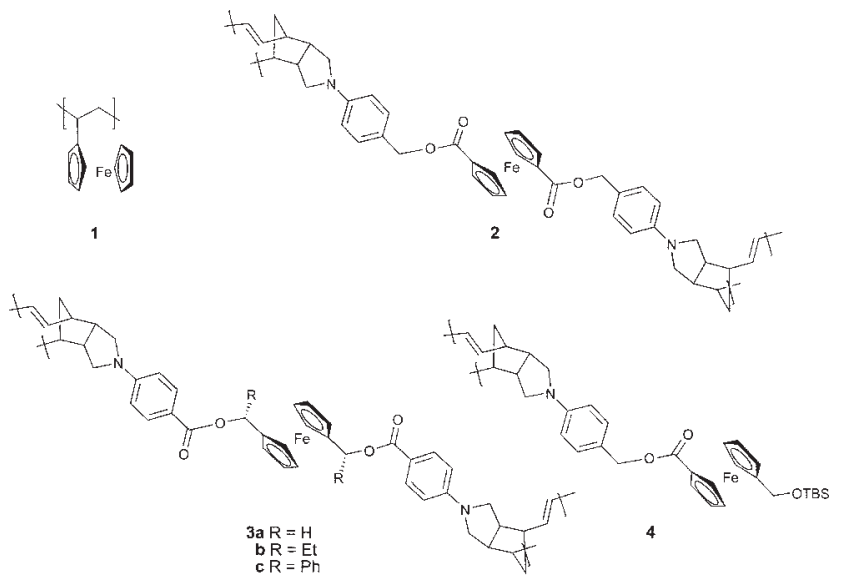

Results of the cyclic voltammetry of $\mathbf{3 a} \mathbf{a}-\mathbf{c}$ are summarised in Table 1, and the cyclic voltammograms are shown in Fig. 1. A redox pair around $100 \mathrm{mV}$ (with reference to the ferroceneferrocenium couple) was assigned to the redox of ferrocene moieties in 3. The irreversible electrochemical oxidation of the aminobenzoate moiety in $\mathbf{3}$ gave an anodic peak around $650 \mathrm{mV}$. As shown in Table 1 , the $i_{\mathrm{pc}} / i_{\mathrm{pa}}$ ratios were close to unity (where $i_{\mathrm{pc}}$ and $i_{\mathrm{pa}}$ are the cathodic and anodic peak currents, respectively) indicating that the redox behaviour of the ferrocene moieties in $\mathbf{3}$ would be reversible (Fig. 1, solid line).

In order to investigate the oxidation characteristics of ferrocene moieties in 3, a fixed potential at $350 \mathrm{mV}$ was employed for bulk electrolysis. The results are listed in Table 2. It is interesting to note that about $70 \%$ of ferrocene moieties in $\mathbf{3}$ were oxidised. When single stranded polymer $\mathbf{4}$ was used for the electrolysis

Table 1 Cyclic voltammetric results of 3 in $\mathrm{CH}_{2} \mathrm{Cl}_{2}$ containing $0.1 \mathrm{M}$ $\mathrm{Bu}_{4} \mathrm{NPF}_{6}$

\begin{tabular}{lllll}
\hline Compound & $E_{\mathrm{pa}}{ }^{a} / \mathrm{V}$ & $E_{\mathrm{pc}}{ }^{a} / \mathrm{V}$ & $\Delta E_{\mathrm{p}} / \mathrm{V}$ & $i_{\mathrm{pc}} / i_{\mathrm{pa}}$ \\
\hline 3a & 0.112 & 0.078 & 0.034 & 0.84 \\
3b & 0.062 & 0.022 & 0.040 & 0.94 \\
3c & 0.147 & 0.071 & 0.076 & 0.99
\end{tabular}

${ }^{a}$ Peak potentials determined from cyclic voltammograms with potential scans involving the redox reactions of the ferrocene moieties only. The electrochemical potentials are reported relative to the ferrocene/ ferrocenium redox couple measured after every experiment. 

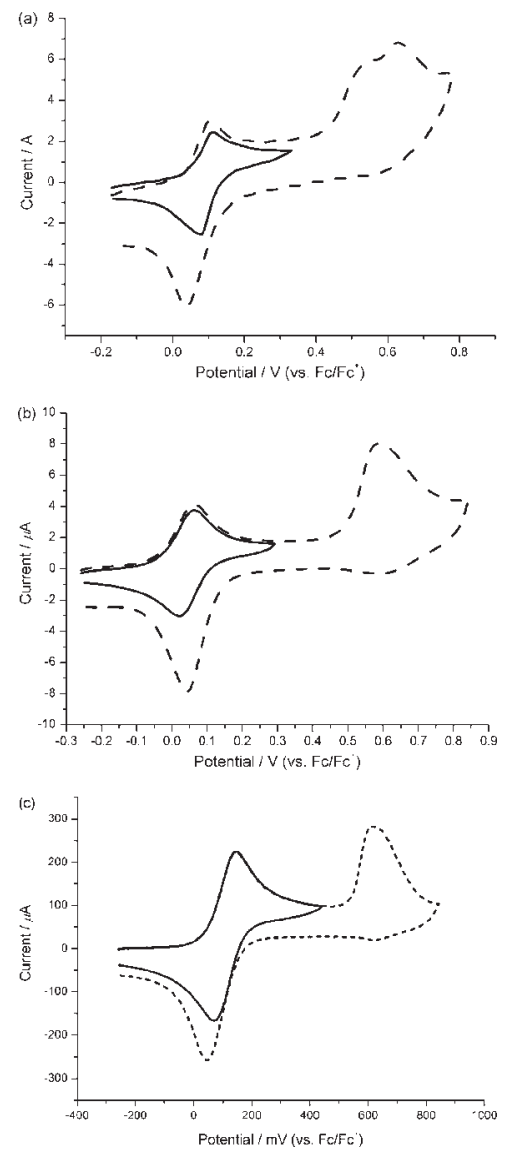

Fig. 1 Cyclic voltammograms of (a) $\mathbf{3 a}$, (b) $\mathbf{3 b}$ and (c) $\mathbf{3} \mathbf{c}$ in $\mathrm{CH}_{2} \mathrm{Cl}_{2}$ containing $0.1 \mathrm{M} \mathrm{Bu}_{4} \mathrm{NPF}_{6}$. Scan rate $=100 \mathrm{mV} \mathrm{s}^{-1}$. The adsorption of oxidised polymers onto the electrode surface may result in the appearance of larger $i_{\mathrm{pc}}$ of the ferrocene moieties.

experiment, a similar percentage of ferrocene groups were oxidised. It is noteworthy that the pending ferrocene moieties in $\mathbf{4}$ have been shown to align essentially in the same manner as those of 3 . As shown in Table 1, differences of some of the redox peak potentials of these polymers, $\Delta E_{\mathrm{p}}$, were somewhat smaller than $59 \mathrm{mV}$. It is noteworthy that each of these apparent redox pairs would represent a summation of multiple electron transfer processes in each of these polymers. Because of such strong overlaps among individual redox processes, the apparent $\Delta E_{\mathrm{p}}$, of these polymers might appear to be smaller than $59 \mathrm{mV}$.

Table 2 Electron transfer ratios of the ferrocene moieties in the polymers estimated by bulk-electrolysis (BE) experiments and by chronoamperometry at a microelectrode (CM)

\begin{tabular}{llrcl}
\hline & & \multicolumn{2}{c}{ Electron transfer ratio $^{a}(\%)$} \\
\cline { 3 - 5 } & $M_{\mathrm{n}}$ & $n_{\mathrm{p}}$ & $\mathrm{BE}$ & $\mathrm{CM}$ \\
\hline 3a & 13700 & 19 & 75 & - \\
3b & 31100 & 40 & 68 & 70 \\
3c & 31200 & 36 & 69 & $-\overline{74}$ \\
3c & 37600 & 43 & - & $-\overline{70}$ \\
$\mathbf{4}$ & 1700 & 8 & 105 & 69
\end{tabular}

${ }^{a}$ The percentage of ferrocene units in a polymer that undergo electron transfer during the bulk-electrolysis process.
It is well documented that the ferrocene moieties in $\mathbf{1}$ were fully oxidised under similar conditions. ${ }^{1}$ We have reexamined the octamer 1 and found complete oxidation of the ferrocene moieties. Apparently, the relative orientation of ferrocene moieties in $\mathbf{1}$ and those in $\mathbf{3}$ and $\mathbf{4}$ would be very different.

Electron transfer numbers of the ferrocene moieties in $\mathbf{3 b}, \mathbf{3 c}$ and 4 were also examined by the chronoamperometric method on an microelectrode, ${ }^{1 a}$ and the results are also summarised in Table 2. On the average, there were around $70 \%$ of the total ferrocene units in these polymers were electrochemically oxidised during a potential-step process. The results obtained by this protocol were consistent with those acquired by bulkelectrolysis experiments described above.

The oxidised $\mathbf{3 c}$ was subjected to magnetic measurements, and the magnetic susceptibilities at different temperatures thus obtained are shown in Fig. 2. The molar magnetic susceptibility, $\chi_{\mathrm{M}}$, was calculated based on the contribution of ten ferrocene-containing monomers; the spin only $\chi_{\mathrm{M}} T$ value of one unpaired electron $(S=1 / 2)$ should be $0.375 \mathrm{~cm}^{3} \mathrm{~K} \mathrm{~mol}^{-1.8}$. Accordingly, the measured $\chi_{\mathrm{M}} T$ value at $295 \mathrm{~K}$ of $2.62 \mathrm{~cm}^{3} \mathrm{~K}$ $\mathrm{mol}^{-1}$ would suggest that roughly seven ferrocene out of every 10 monomers being oxidised. In other words, there would be $70 \%$ of the ferrocenium moieties, which is supposed to have one unpaired electron, in the oxidised 3c. This result agreed nicely with those from the electrochemical measurements. Moreover, the $\chi_{\mathrm{M}} T$ increases with temperature from 2 to $300 \mathrm{~K}$, which indicates the strong antiferromagnetic coupling among the oxidised monomers. ${ }^{9}$

The oxidation behaviours of $\mathbf{3}$ and $\mathbf{4}$ are apparently very different from those of $\mathbf{1}^{1}$ and the sulfur- or silylene-spaced ferrocene copolymers. ${ }^{2}$ The structural uniqueness of $\mathbf{3}$ and $\mathbf{4}$ may play a pivotal role in this electrochemical oxidation process. That $70 \%$ of the ferrocene moieties in $\mathbf{3}$ and $\mathbf{4}$ were oxidised suggest that each of the neighbouring monomeric units may strongly couple with each other, which can be evidenced by the antiferromagnetic interactions observed in magnetic susceptibility measurements. However, due to the electrostatic repulsion, the ferrocene linkers in $\mathbf{3}$ and $\mathbf{4}$ cannot be fully oxidised. It is noteworthy that the oxidised form of $\mathbf{3}$ and $\mathbf{4}$ appeared to be fairly stable under ambient conditions.

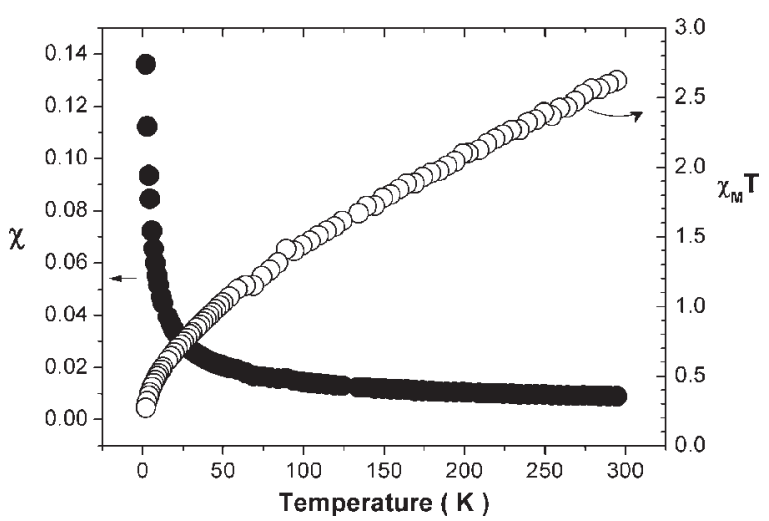

Fig. 2 Magnetic susceptibility of the oxidised $\mathbf{3 c}$ as a function of temperature. The solid $(\chi)$ and open circle $\left(\chi_{M} T\right)$ are experimental data based on ten monomeric units after correction on diamagnetism and normalization on the applied magnetic field. 
Table 3 Hirshfeld charge analysis on $\mathbf{5}$ and $\mathbf{5}^{n+}(n=4-6)$

\begin{tabular}{lllllll}
\hline Compound & $\mathrm{Fe}$ & $\mathrm{Fe}$ & $\mathrm{Fe}$ & $\mathrm{Fe}$ & $\mathrm{Fe}$ & $\mathrm{Fe}$ \\
\hline $\mathbf{5}$ & 0.0814 & 0.0867 & 0.0866 & 0.0866 & 0.0867 & 0.0814 \\
$\mathbf{5}^{4+}$ & 1.2577 & 0.3469 & 0.1252 & 0.1252 & 0.3469 & 1.2577 \\
$\mathbf{5}^{5+}$ & 1.6106 & 0.3590 & 0.1255 & 0.1255 & 0.3590 & 1.6106 \\
$\mathbf{5}^{6+}$ & 1.9636 & 0.3710 & 0.1259 & 0.1259 & 0.3710 & 1.9636 \\
\hline
\end{tabular}

As depicted earlier, the ferrocene moieties in $\mathbf{3}$ may be aligned coherently in essentially eclipsed manner with the spacing between neighbouring ferrocene moieties about $5.5 \AA$. In order to realise the charge distribution of the oxidised form of 3, DFT calculations were undertaken on a model having six ferrocenes aligned linearly with a separation of $5.5 \AA$ between the neighbouring ferrocenes, 5. Each of the ferrocene moieties was assumed in $D_{5 \mathrm{~h}}$ point group and a $C_{2 \mathrm{v}}$ symmetry was imposed on $\mathbf{5} . \ddagger$ The calculation of neutral $\mathbf{5}$ was carried out in the restricted closed shell level. Four to six electrons were then removed from the HOMO to HOMO-2 of the neutral 5 to give the corresponding oxidised $\mathbf{5}^{n+}(n=$ 4-6) and the charge densities on each ferrocene moiety in these ions were obtained. $\ddagger$ The Hirshfeld charge analysis ${ }^{10}$ at $\mathrm{Fe}$ atoms on $\mathbf{5}$ and $\mathbf{5}^{n+}$ are summarised in Table 3. It is interesting to note that removal of electrons from $\mathbf{5}$ would yield uneven charge distribution among Fe atoms in oxidised forms of $\mathbf{5}^{n+}$. The majority of the charge is located at the terminal ferrocene moieties with minor charge resided at the interim ferrocene moieties. These results indicate the charges are delocalised and accumulated at the terminal ones to minimise the overall Coulombic interactions. The molecular orbital diagrams for HOMO to HOMO -5 and LUMO to LUMO +1 are provided in the ESI. $\dagger$ Extensive oxidation up to the removal of five and six electrons from $\mathbf{5}$ may, however, result in too much charge localised at the terminal ferrocene moieties which may somewhat destabilise the system.
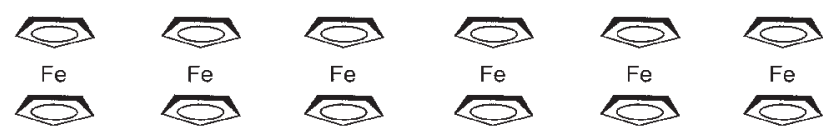

5

In summary, we have demonstrated the unique electrochemical properties of the ferrocene-containing double stranded bispolynorbornenes 3 and related polymer $\mathbf{4}$. The antiferromagnetic character of the stable oxidised $\mathbf{3 c}$ suggested strong coupling between the neighbouring ferrocene/ferrocenium moieties. The unusual structural feature and distinctive redox characteristics of these polymers may be useful for future applications in catalysis as well as in optoelectronic applications.

This work was supported by the National Science Council and the National Taiwan University of the Republic of China. We thank Professor Allen J. Bard for helpful discussions and $\mathrm{NCHC}$ for the support on the hardware and software for molecular orbital calculations.

\section{Notes and references}

$\ddagger$ The calculations were carried out by Amsterdam Density Functional $2006.01(\mathrm{ADF})^{11}$ with the generalised gradient approximation (GGA). The corrections of LDA and GGA parts were taken from $\mathrm{VWN}^{12}$ and BP. ${ }^{13,14}$ The SCALAR ZORA was used for relativistic corrections. The triple zeta with two polarization function, TZ2P, Slater type basis sets were used for all atoms.

1 (a) J. B. Flanagan, S. Margel, A. J. Bard and F. Anson, J. Am. Chem. Soc., 1978, 100, 4248; (b) P. J. Peerce and A. J. Bard, J. Electroanal. Chem., 1980, 114, 89; (c) D. Albagli, G. Bazan, M. S. Wrighton and R. R. Schrock, J. Am. Chem. Soc., 1992, 114, 4150; (d) M. Morán, C. M. Casado, I. Cuadrado and J. Losada, Organometallics, 1994, 12, 4327; (e) A. L. Crumbliss, D. Cooke, J. Castillo and O. Wisian-Neilson, Inorg. Chem., 1994, 32, 6088.

2 (a) P. F. Brandt and T. B. Rauchfuss, J. Am. Chem. Soc., 1992, 114, 1926; (b) D. A. Foucher, B. Z. Tang and I. Manners, J. Am. Chem. Soc., 1992, 114, 6246; (c) J. M. Nelson, H. Rengel and I. Manners, J. Am. Chem. Soc., 1994, 115, 7035; (d) M. T. Nguyen, A. F. Diaz, V. V. Dement'ev and K. H. Pannell, Chem. Mater., 1994, 5, 1389; (e) D. L. Compton and T. B. Rauchfuss, Organometallics, 1994, 13, 4367; (f) C. M. Casado, I. Cuadrado, M. Morán, B. Alonso, B. García, B. González and J. Losada, Coord. Chem. Rev., 1999, 185-186, 53; $(g)$ G. E. Southard and M. D. Curtis, Organometallics, 2001, 20, 508; $(h)$ R. W. Heo, J.-S. Park, J. T. Goodson, G. C. Claudio, M. Takenaga, T. A. Albright and T. R. Lee, Tetrahedron, 2004, 60, 7225; (i) P. Park, A. J. Lough and D. A. Foucher, Macromolecules, 2002, 35, 3810; (j) X.-J. Wang, L. Wang, J.-J. Wang and T. Chen, Electrochim. Acta, 2007, 52, 3941 .

3 (a) I. Cuadrado, C. M. Casado, B. Alonso, M. Morán, J. Losada and V. Belsky, J. Am. Chem. Soc., 1997, 119, 7613; (b) S. Nalte, J. Ruiz, J.-C. Blais and D. Astruc, Chem. Commun., 2000, 417; (c) S. Nalte, J. Ruiz, V. Sartor, R. Navarro, J.-C. Blais and D. Astruc, Chem.-Eur. J., 2000, 6, 2544.

4 (a) P. Ceroni, V. Vicinelli, M. Maestri, V. Balzani, M. Gorka, S.-K. Lee, P. Dragut and F. Vögtle, Collect. Czech. Chem. Commun., 2004, 68, 1541; (b) F. Marchioni, M. Venturi, P. Ceroni, V. Balzani, M. Belohradsky, A. M. Elizarov, H.-R. Tseng and J. F. Stoddart, Chem.-Eur. J., 2004, 10, 6361; (c) K. Krishnamoorthy, R. R. Dasari, A. Nantalaksakul and S. Thayumanavan, Chem. Commun., 2007, 739.

5 (a) H.-C. Yang, S.-Y. Lin, H.-C. Yang, C.-L. Lin, L. Tsai, S.-L. Huang, I.-W. P. Chen, C.-h. Chen, B.-Y. Jin and T.-Y. Luh, Angew. Chem., Int.Ed., 2006, 45, 726; (b) N.-T. Lin, S.-Y. Lin, S.-L. Lee, C.-h. Chen, C.-H. Hsu, L. P. Hwang, Z.-Y. Xie, C.-H. Chen, S.-L. Huang and T.-Y. Luh, Angew. Chem., Int. Ed., 2007, 46, 4481.

6 W.-Y. Lin, M. G. Murugesh, S. Sudhakar, H.-C. Yang, H. Tai, C.-S. Chang, Y.-H. Liu, Y. Wang, I.-W. P. Chen, C.-h. Chen and T.-Y. Luh, Chem.-Eur. J., 2006, 12, 324.

7 Y.-Y. Lai, N.-T. Lin, Y.-H. Liu, Y. Wang and T.-Y. Luh, Tetrahedron, 2007, 63, 6051.

8 O. Kahn, Molecular Magnetism, VCH, New York, 1993.

9 For a precedented antiferromagnetic linear single-stranded ferrocene-containing polymer, see: J. M. Nelson, P. Nguyen, R. Petersen, H. Rengel, P. M. Macdonald, A. J. Lough, I. Manners, N. P. Raju, J. E. Greedan, S. Barlow and D. O'Hare, Chem.-Eur. J., 1997, 3, 573.

10 F. L. Hirshfeld, Theor. Chim. Acta, 1977, 44, 129.

11 (a) G. te Velde, F. M. Bickelhaupt, S. J. A. van Gisbergen, C. F. Guerra, E. J. Baerends, J. G. Snijders and T. Ziegler, J. Comput. Chem., 2001, 22, 931; (b) C. Fonseca Guerra, J. G. Snijders, G. te Velde and E. J. Baerends, Theor. Chim. Acta, 1998, 99, 391.

12 S. H. Vosko, L. Wilk and M. Nusair, Can. J. Phys., 1980, 58, 1200.

13 A. D. Becke, Phys. Rev. A, 1988, 38, 3098.

14 J. P. Perdew and W. Yang, Phys. Rev. B, 1986, 33, 8800. 